\title{
Post-operative assessment in Deep Brain Stimulation based on Multimodal images: Registration Workflow and Validation
}

\author{
Florent Lalys ${ }^{1,2,3}$, Claire Haegelen ${ }^{1,2,3,4}$, Alexandre Abadie ${ }^{1,2,3}$, Pierre Jannin ${ }^{1,2,3}$ \\ 1 INSERM, U746, Faculty of Medicine CS 34317, F-35043 Rennes, France \\ 2 INRIA, VisAGeS Unit/Project, F-35042 Rennes, France \\ ${ }^{3}$ University of Rennes I, CNRS, UMR 6074, IRISA, F-35042 Rennes, France \\ ${ }^{4}$ Department of Neurosurgery, Pontchaillou University Hospital, F-35043 Rennes, France
}

\begin{abstract}
Object

Movement disorders in Parkinson disease patients may require functional surgery, when medical therapy isn't effective. In Deep Brain Stimulation (DBS) electrodes are implanted within the brain to stimulate deep structures such as SubThalamic Nucleus (STN). This paper describes successive steps for constructing a digital Atlas gathering patient's location of electrodes and contacts for post operative assessment.

\section{Materials and Method}

12 patients who had undergone bilateral STN DBS have participated to the study. Contacts on post-operative CT scans were automatically localized, based on black artefacts. For each patient, post operative CT images were rigidly registered to pre operative MR images. Then, pre operative MR images were registered to a MR template (super-resolution Collin27 average MRI template). This last registration was the combination of global affine, local affine and local non linear registrations, respectively. Four different studies were performed in order to validate the MR patient to template registration process, based on anatomical landmarks and clinical scores (i.e., Unified Parkinson's disease rating Scale). Visualisation software was developed for displaying into the template images the stimulated contacts represented as cylinders with a colour code related to the improvement of the UPDRS.
\end{abstract}

\section{Results}

The automatic contact localization algorithm was successful for all the patients. Validation studies for the registration process gave a placement error of $1.4+/-0.2 \mathrm{~mm}$ and coherence with UPDRS scores.

\section{Conclusion}

The developed visualization tool allows post-operative assessment for previous interventions. Correlation with additional clinical scores will certainly permit to learn more about DBS and to better understand clinical side-effects.

\section{KEYWORDS}

Deep brain stimulation, Post-operative control, neurosurgery, registration, targeting accuracy 


\section{1 - INTRODUCTION}

The subthalamic nucleus (STN) became the mean target of high-frequency deep brain stimulation (DBS) in patients with Parkinson's disease (PD) and severe disabled symptoms [1-5]. While symptoms related to PD can be treated without surgical intervention, it remains patients for which medical therapy is not effective. The targeting of the STN is based on anatomic, imaging and statistical data obtained on anatomic and prospective clinical studies. Instead clinical data aren't often used yet to localize optimal target, teams worked on successful implantations where post-operative effects are satisfactory and side effects minimum. They can with these results find the best localization related to tremor, bradykinesia or rigidity pathologies, and provide the surgical team with a complete comprehensive neurosurgical system. New MR imaging protocols enable visualization of brain structures. But for dedicated clinical applications such as DBS, a more accurate localization requires the use of Atlases, such as histological [6,7] or high-resolution [8,9] ones. This paper describes the image processing steps for constructing a digital Atlas gathering patient's location of electrode contacts and validation studies of the registration aspect. UPDRS (Unified Parkinson's disease rating Scale [10]) scores will be integrated in the Atlas to correlate position of stimulated contacts with clinical data.

\section{2 - MATERIALS AND METHODS}

\subsection{Patients}

Twelve patients (8 male, 4 female, mean age 55 +/- 8 years) with idiopathic Parkinson's diseases had STN DBS according to selected inclusion criteria [1,2].

\subsection{Images}

MRI, even with the progress in imaging, is the source of non-predicable distortion, making difficult the possibility of visualizing deep brain structures during the procedure of targeting. Matching pre-operative CT with MRI allows overcoming such issue. Therefore, the geometry accuracy of the CT is preserved, and the structures can be identified with the MRI. Such technique is used here. Patients had pre-operative 3-T T1-weighted MR (1 mm x $1 \mathrm{~mm}$ x $1 \mathrm{~mm}$, Philips Medical Systems), pre and post-operative CT scans $(0.44 \mathrm{~mm}$ x $0.44 \mathrm{~mm}$ x $0.6 \mathrm{~mm}$ in post-operative acquisitions and $0.5 \mathrm{~mm} \times 0.5 \mathrm{~mm} \times 0.6 \mathrm{~mm}$ in pre-operative acquisitions, GE Healthcare VCT 64). Thirty-five slices of preoperative T2-weighted images involving basal ganglia were also acquired for helping the STN targeting.

Images Pre-Processing: Pre and post-operative CT, T1- and T2-weighted MR images were denoised with the Non-local means algorithm [11]. A bias correction algorithm [12] based on intensity values was also applied on T1 and T2 MR images.

\subsection{Clinical procedure}

Pre-operative planning was performed with the Stealthstation planning software (Medtronic, Minneapolis, Minnesota, USA), loading images, registering them together and proposing a 3D visualisation interface to define the target localisation in the Anterior and Posterior Commisures (AC-PC). T1, T2 and CT images were first co-register, and secondly experts localized manually AC-PC coordinates, the midsagittal and entry points on the MR images. The Talairach [13] Atlas was then displayed, where experts finally localized the optimal target position based on the anatomic information of the patient. Coordinates of the target were automatically transformed into CA-CP coordinate system, in order to reach the optimal position of contact with the quadripolar electrode (DBS 3389, Medtronic) during the surgical procedure.

Surgical procedure: Stereotactic electrode implantation (Leksell, USA) is performed under local anaesthesia in a 1 day procedure. Both preoperative T1/T2-weighted MRI and CT are obtained early in the morning, following by the target localization explained above.

The point calculated before the implantation is used as an initial position that has to be refined intra-operatively. A few causes of discrepancy between chosen target and the final implant might appear, such as the brain shift [14,15] and the stereotactic surgical placement errors. The first one is due to the opening of the brain, but it is assumed that there are no significant errors which can causes real perturbation in our results. The second one is due to the mechanic placement errors. In spite of a submillimetric precision in graduation the frame causes a loss of accuracy that is no negligible. To take into account these different errors an X-ray control is done during the surgical procedure, helping the neurosurgeons to place correctly the electrode. In order to reach the optimal placement, a phase of intra-operative electrophysiological 
exploration is also performed. Two neurologists test the clinical effects with different settings for each contact, when the patient is still under local anaesthesia. Changing frequency, voltage, contact stimulated and electrodes trajectory they finally keep the best settings, knowing that these one might be change post-operatively as Parkinson's pathologies are often evolving and need readjustments. Of course this choice is also guide by the side effects which should be as minimum as possible. At every permutation of settings, a small time is necessary to allow the clinical effects of patient's pathology to come back. When all the test are finished, the surgeon anchor the electrode to the skull, and if the surgical procedure is a bilateral DBS implantation the second electrode is then subjected to the same steps. Typically a monolateral implantation is performed when patient's tremor disorders are located to only one side (in that case electrode is implanted in the other side of the brain). Instead of this step of intra-operative programming, clinical effects may take several weeks to appear, which complicate the task of the neurologists in finding the optimal settings.

\subsection{Automatic contact localization}

The spatial coordinates of the contacts for each electrode were computed post-operatively from CT images. Their positions were determined based on the black artefacts created on the CT. Recent works [16] on electro-magnetic effects showed that artefacts appearing on images corresponded to the center of the real contact. This segmentation task was automatically realized, with at first an intensity threshold, followed by a search of artefacts on the image and constraints on neighbourhood. Usually just two or three contacts can be identified directly on the image because of the resolution of the slices, which caused loss of information. To find the last contact, it was necessary to extrapolate the points already found with the original electrode settings, such as the distance of a contact $(1.5 \mathrm{~mm})$ or the distance between each $(0.5$ $\mathrm{mm}$ or $1.5 \mathrm{~mm})$. On every new post-operative data this automatic procedure was performed and gave all contacts localizations.

\subsection{Contact representation}

With the method mentioned previously, active contacts are represented by a point corresponding to the center of the contact. Some previous works estimated the height of the contact [17,18], while others [19] considered all the structures involved in the stimulation, which means considering the contacts lying within the different structures. For the representation in the atlas, we decided to take into account all the geometry of a contact, modelled by a cylinder (length: $1.5 \mathrm{~mm}$, diameter: $1 \mathrm{~mm}$ ) (Fig 1). On the other hand, for the database we kept the representation as a point, in computing the centroid of the projected artefacts.

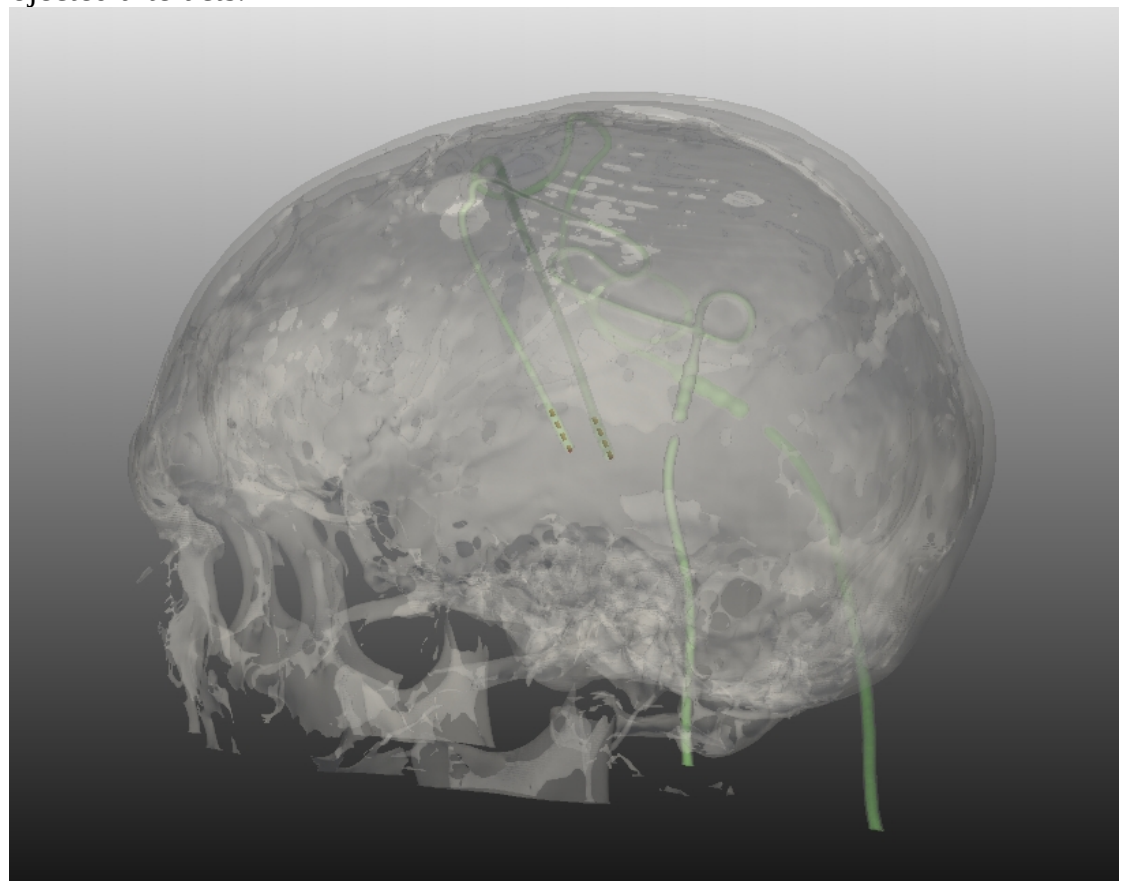

Fig 1: Segmentation of two electrodes, their contacts and the skull of a patient with STN DBS for Parkinson's disease. 


\subsection{Registration}

We used the state of the art of linear/non-linear registration algorithm. We decided to use the technique already described by Sanchez [20], which showed that the best patient images to Atlas registration in the context of DBS was a global linear image to Atlas registration with a mask on deep structures, following by a non-linear local registration with either Demons or BSplines algorithm. We added CT to MRI registration step.

Here is the registration procedure proposed for each subject (Fig 2). The post-operative CT was first linearly registered to the pre-operative MRI, creating a 3D transformation matrix. The affine MR-to-atlas transformation was then computed. After the global warping, a local affine registration was computed on a region of interest including the deep brain structures. All the linear transformations were executed with the Newuoa optimization [21]. The final step included a non-linear local algorithm called Demons [www.itk.org], which estimated a 3D deformation field between a source volume and a target volume. Non-linear was essential to take into account the difference of anatomy between each patient. We finally obtained a global matrix including the four linear transformations, and a deformation field corresponding to the non-linear one. With this procedure the contacts positions could be warp in the common Colin27 template and it allows visualization of contacts in deep brain structures of the Template. On the contrary, it also allows register the possible optimal electrode contacts from the Atlas to the pre-operative acquired data by an inverse of the original transformation, since linear and non-linear algorithms are both bijective transformations.

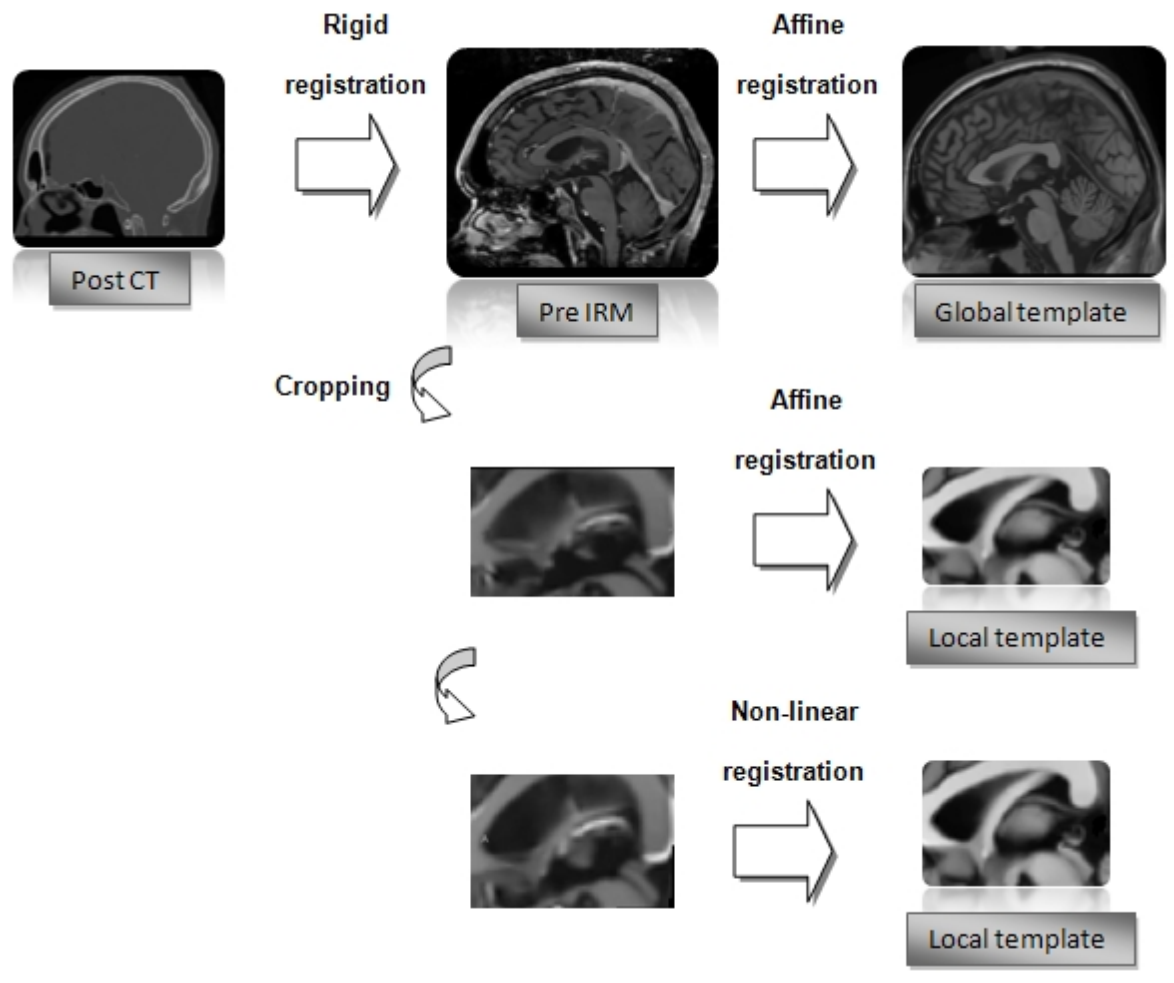

Fig 2: Registration Workflow between post-operative CT and the MR template

\subsection{Registration Validation}

Four different validation studies of the registration workflow were performed with increased clinical meaning, considering that rigid intra-patient CT to MR registration was almost perfect. The first validation study used statistic tools to evaluate the similarity of two coregistered images, in order to compare several input parameters of our method: "global" vs. "local" registration, the choice of non-linear algorithm and the choice of specific template. The performance criterion used was the intensity based correlation, as non-linear algorithms in our procedure are based on mutual 
information. Mathematical approach permits to characterize the intrinsic performance of a method to have first results on best settings to keep. The second validation got closer to the clinical context and was based on 6 anatomical landmarks defined by an expert and identified on each patient registered MRI and on the MR template. Euclidean distance for each landmark defined in both images was computed on the 12 patients, in order to get the global misplacement error. The third validation study was based on Talairach results [13], which mentioned that deep brain structures have more or less the same coordinates in images if those ones are taking into a reference space called Talairach (based on AC-PC). Thus contacts coordinates were computed at every step of our procedure in defining AC-PC on each reference, from rigid to non-linear registration. Then the Euclidean distance of the contact coordinates between each reference was computed. The reference in that case was based on clinical a priori. With that method we ensured that no significant errors existed and that our final results were valuable. The final validation permitted to assess our method as regards to patient outcome. An expert segmented the STN to ensure that stimulated contacts belonged to the structure and then simple correlations were made between the improved motor score of the Unified Parkinson's Disease Rating Scale (UPDRS) part III (which has been evaluated 3 pre- and 3 months-postoperatively) and the contact's position to assess the patient outcome efficacy $[17,22]$.

\subsection{Cluster}

We assigned to each patient a colour code, related to the improved score of the UPDRS III. We considered the percentage of improvement between both, from $0 \%$ to $100 \%$. Then we separated five clusters from these scores and we displayed the contacts on the template with a corresponding colour.

\section{3 - RESULTS}

\subsection{Registration Validation}

Our registration workflow studies gave us results on best methods and algorithms to keep. According to Tab 1, the local method widely gives best images warping, with a significant difference for mutual information. This study shows that local registration is fundamental to place correctly the deep structures.

\begin{tabular}{ccccc} 
& \multicolumn{2}{c}{ Mutual Information } & \multicolumn{2}{c}{ Correlation } \\
\cline { 2 - 5 } & 1st method & 2nd method & 1st method & 2nd method \\
\hline average on 10 patients & $81,11 \%$ & $68,01 \%$ & $82,19 \%$ & $80,46 \%$ \\
\hline
\end{tabular}

Tab 1 : Mutual information and correlation between the final images registered and the Template, using with the first method a local registration, and with the second a global registration.

We consider for granted, according to [20], that the Demons method is the most effectiveness in DBS non-linear registration. Table 2 shows that firstly the super-resolution Colin27 is better than the original, and secondly that the SymetricForces algorithm is more accurate than the Diffeomorphic one. These conclusions can be drag out from both correlation and mutual information calculation.

\begin{tabular}{|c|c|c|c|c|c|c|c|c|}
\hline & \multicolumn{4}{|c|}{ Mutual Information } & \multicolumn{4}{|c|}{ Correlation } \\
\hline & \multicolumn{2}{|c|}{ Original Colin27 } & \multicolumn{2}{|c|}{ Super-resolution Colin27 } & \multicolumn{2}{|c|}{ Original Colin27 } & \multicolumn{2}{|c|}{ Super-resolution Colin27 } \\
\hline & Diffeomorphic & SymmetricForces & Diffeomorphic & SymmetricForces & Diffeomorphic & SymmetricForces & Diffeomorphic & SymmetricForces \\
\hline average on 10 patients & $69,53 \%$ & $89,77 \%$ & $96,52 \%$ & $99,99 \%$ & $81,26 \%$ & $84,68 \%$ & $88,43 \%$ & $89,44 \%$ \\
\hline
\end{tabular}

Tab 2 : Mutual information and correlation between the final images registered and the Template, using either the original Colin27 or the super-resolution Colin27, and using the Diffeomorphic algorithm and the SymetricForces one respectively.

Table 3 shows the global registration errors on the Template, using first linear registration, and second adding the nonlinear one. Statistics are also display in Fig 3. 


\begin{tabular}{cccccccc} 
Point & CA & CP & Iter of Sylvius & Chiasma opticum & Mamillary bodies & Left Tractus optici & Average \\
\hline Linear & 1,26 & 1,29 & 1,51 & 2,21 & 1,6 & 2,1 \\
\hline Linear + Non-linear & 1,17 & 0,71 & 1,03 & 1,66 & 1,89 & 1,9 \\
\hline
\end{tabular}

Tab 3 : Euclidean distance between landmarks on the linear registered MRI compare to the Template, and on the non-linear registered MRI compare to the Template.

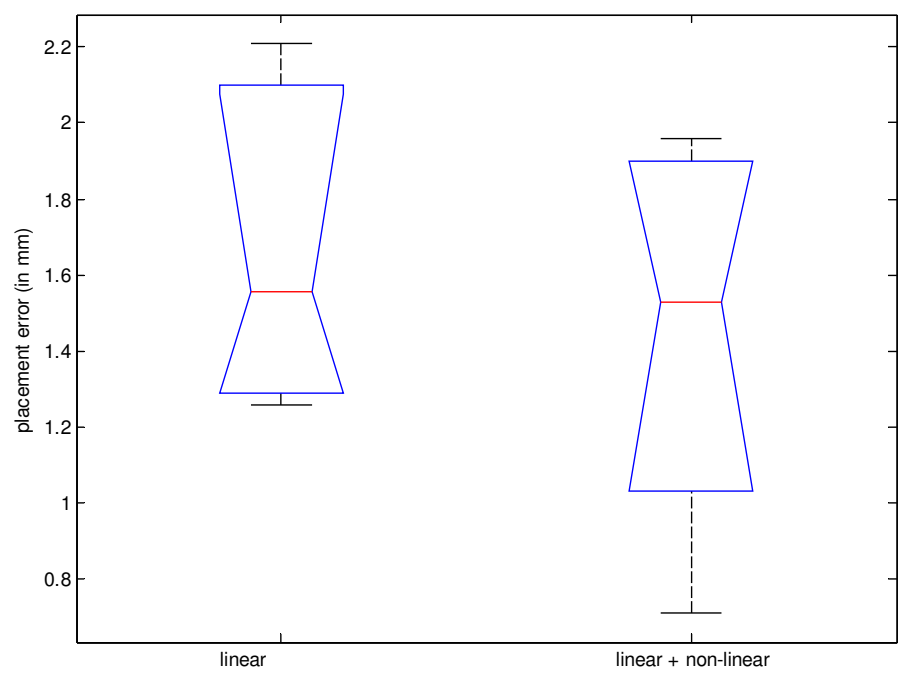

Fig 3 : Anova1 (MATLAB) performed on the previous study, for placement error coming from linear registration, and from linear + non-linear registration.

We obtained, with the same ten patient's study, a placement error of $1.44 \mathrm{~mm}$ compare to $1.66 \mathrm{~mm}$ with a single linear method. Even if the two averages are close, Anova shows that the majority of values for the non-linear registration are under $1.5 \mathrm{~mm}$. The best warping came from AC and PC so central deep structures seem to be well-warped.

The contacts coordinates in AC-PC from image to image were coherent $(3.0 \mathrm{~mm}+/-1.0 \mathrm{~mm})$ and motors score correlated with anatomical coordinates have shown that every stimulated contact belong the STN. Fig 4 shows four electrodes (from two patients) and their contacts, represented by cylinder in the Colin27 space. 

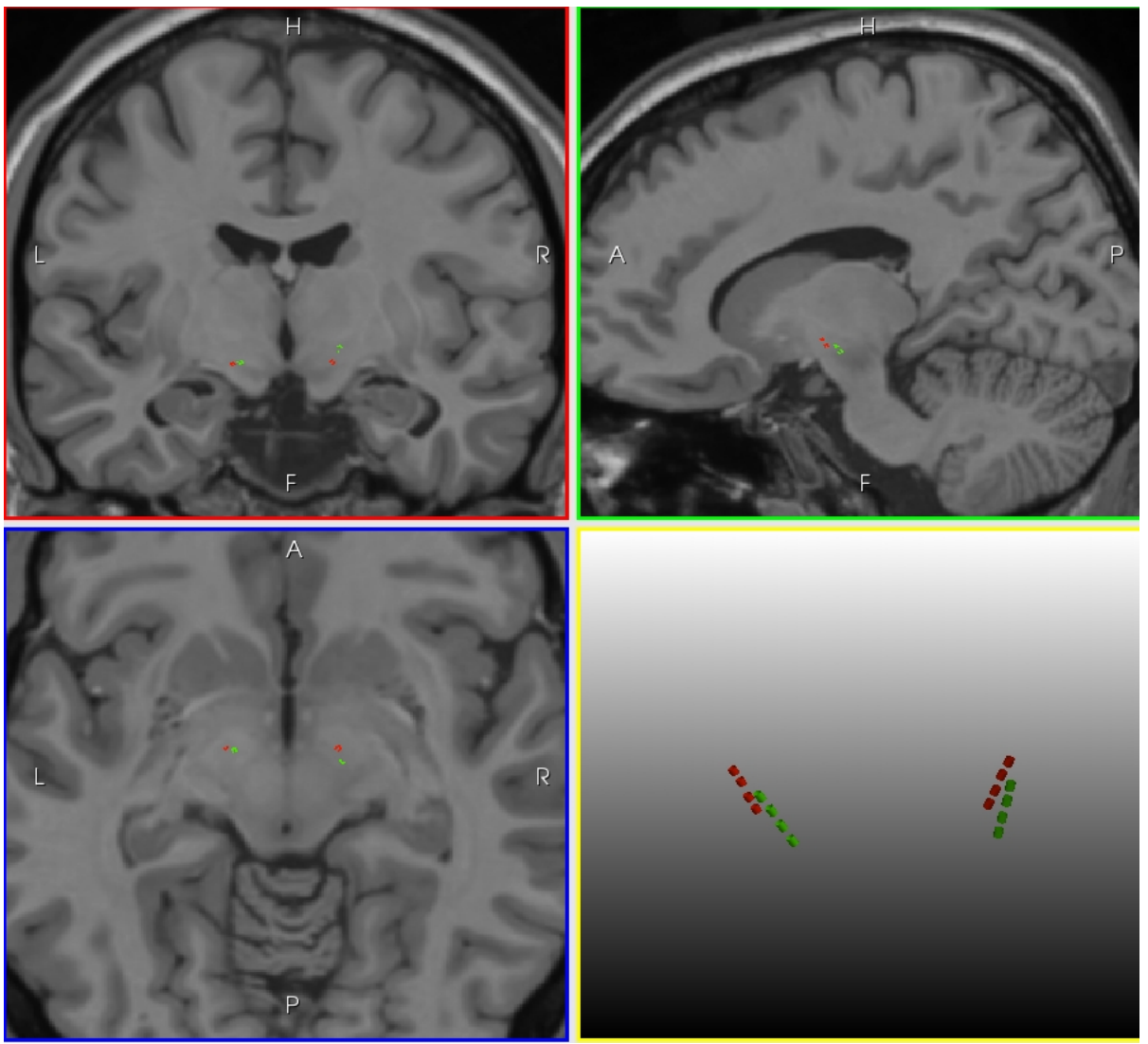

Fig 4: Representation of four electrodes (implanted in the STN) from two patients in the Colin27 space after registration.

\subsection{Cluster}

The mean pre-operative UPDRS III without medication was $29.3+/-6.1$. The post-operative one without medication with stimulation was $13.1+/-4.1$. Recent publications have permitted to find the most effective stimulation point in Parkinson's diseases. The best stimulation point would be the dorsal area of the STN.

\subsection{Database}

Contacts localizations, following by the registration procedure permits a global display of previous surgical intervention in a common space. The database contains now all coordinates from previous operation, with corresponding patient images and clinical scores. Neurosurgeons have a first computer-aided-placement tool.

\section{4 - DISCUSSION}

\subsection{Atlas}

Use of digitalized atlases is important in DBS, for being able to help anatomical targeting. A digitalized Atlas can give information at the histological level which is impossible with actual imaging techniques. With this help, subcortical anatomy can be visible and can be match with every patient anatomy.

Paper-based atlases were originally used for such purposes. Those of Schaltenbrand and Wahren [23], or Talairach and Tournoux [13] have been used with success in various computer aided decision systems. More recently atlases were built from digital images. Digital atlases can be classified according to the number of subjects used for the computation. Multiple subject atlases are mainly built from single acquisition of different control subjects reflecting the population targeting by the clinical study [24,25]. Such multi-subjects templates mainly aim at studying human anatomical or 
functional variability. Being able to merge anatomical or functional differences into a single image may improve the correct alignment before comparison. Single subject atlases aim at reaching the best spatial resolution. Different strategies were followed for defining such templates. The first strategy was the use of co-registered mono or multimodal acquisitions of a single particular subject $[8,9]$ mainly based on MR acquisitions. The second was the combination of images and histological atlases [6,7]. The interest of the first strategy is that the whole clinical acquisition set up is taken into account on healthy and alive control subjects. In opposition to pure histological Templates, MR templates are representing correctly in vivo anatomy of the brain. Moreover, they turn out to be easily feasible, whereas histological ones are complicated or time-cost to produce. These Atlases are constrained by both the resolution and the quality of available imaging technologies.

In the case of DBS, new family of Atlases appear, called probabilistic functional Atlases [26-28]. A large panel of parameters can be integrated in this type of Atlases, such as position of electrodes, stimulation recordings or even electrophysiological recordings. For instance, studies proposed an Atlas integrating intra-operative recordings, which can be match with patient pathologies [29,30]. This technique allows understanding functional organization within deep brain structures. Such fusions of images should be taken with precaution, because differences of anatomy between each patient make difficult the representation of target/contact in a common space. Here we used the super-resolution Colin27 template, which gave us best results compare to a standard Atlas. The impact of this template was significant and it has improved not only the registration quality but also the retrospective visualization. The addition of clinical data has allowed understanding functional organization within deep brain structures. It's certainly a part of our procedure that can be more and more improved, with the help of new imaging technologies (3T machines) and post-processing. The more the Template will be accurate; the better will be the contacts visualization.

\subsection{Accurate localization}

Using conclusions of published works on electro-magnetic contacts effects, we have modelled the signal by a point corresponding to the center of the artefact. For further works, it will be crucial to integrate not only the real electric current that contacts emit but also the influence of stimulation on the biological tissues. The extrapolation created with the artefacts is based on electrode geometry and is therefore robust.

\subsection{Registration validation}

With all studies performed for the assessment, we have completely validated our registration workflow as we have found appropriate methods and algorithms [31]. Rigid registration algorithms provide mostly effectiveness results, whereas non-rigid algorithms have several limits. Various non-rigid algorithms for the DBS studies have been published but it's almost impossible to assess all the kind of approach, due to the difficulty to have access to a ground truth.

In DBS major sources of errors are due to the identification of basal ganglia from the patient specific images. Therefore, during the procedure we have added a local registration involving targeted structure (STN) which has to improve the basal ganglia registration. We are still working on development and improvement of the registration strategy, even though the results from validation studies were correct.

\section{5 - CONCLUSION}

In this paper we have reported on our current progress toward developing a procedure for computer-assisted postoperative assessment of DBS. We principally aimed to have robust and assessed results, using several kind of assessment in registration. Moreover, testing various methods and algorithms, we finally kept the most effective procedure of registration and use it for our study. The extrapolation created with the artefacts is based on electrode geometry and is therefore robust; furthermore if we add constraint on neighbourhood the risk of misplacement is considerably reduced. The integration in the same system of digitized brain Atlas, segmented structures, previous target coordinates of successful implantations and UPDRS score not only permits to provide to the neurosurgeons an help for targeting, but also permits to understand previous interventions which didn’t give satisfactory results. This work yields many flourishing studies in the domain, including further clinical data such as neuro-psychological, life-quality, cognitive or motor criteria. Correlation with these additional clinical scores will certainly permit to learn more about DBS and to better understand clinical side-effects. 


\section{REFERENCES}

[1] Lang AE, Lozano AM, “Parkinson’s Disease”, The New England Journal of Medicine, 339(15), 1044-1053 (1998).

[2] The Deep-Brain Stimulation for Parkinson’s Disease Study Group, “Deep-Brain Stimulation of the Subthalamic Nucleus or the Pars Interna of the Globus Pallidus in Parkinson's Disease”, The New England Journal of Medicine, 345(13), 956-963 (2001).

[3] Rodriguez-Oroz MC, Rodriguez M, Guridi J, Mewes K, Chockkman V, Vitek J, DeLong MR, Obeso JA, "The subthalamic nucleus in parkinson's disease: somatotopic organization and physiological characteristics", Brain, 124, 1777-1790 (2001).

[4] Krack P, Batir A, Van Blercorn N, Chabardes S, Fraix V, Ardouin C, Koudsie A, Dowsey Limousin P, Benazzouz A, LeBas JF, Benabib AL, Pollak P, "Five-Year Follow-up of Bilateral Stimulation of the Subthalamic Nucleus in Advanced Parkinson's Disease”, The New England Journal of Medicine, 349(20), 1925-1934 (2003).

[5] Vayssière N, Vander Gaag N, Cif L, Hemm S, Verdier R, Frerebeau P, Courbes P, "Deep brain stimulation for dystonia confirms a somatotopic organization in the Globus Pallidus Internus”, J Neurosurg, 101(2), 181-188 (2004).

[6] Yelnik J, Bardinet E, Dormont D, Malandain G, Ourselin S, Tandé D, Karachi C, Ayache N, Cornu P, Agid Yves, "A three-dimensional, histological and deformable atlas of the human basal ganglia. I. Atlas construction based on immunohistochemical and MRI data", NeuroImage, 37, 618-638 (2007).

[7] Chakravarty M, Bertrand G, Hodge CP, Sadikot AF, Collins DL, "The creation of a brain atlas for image guided neurosurgery using serial histological data”, NeuroImage, 30, 359-376 (2006).

[8] Aubert-Broche B, Evans AC, Collins L, "A new improved version of the realistic digital brain phantom", NeuroImage, 32, 138-145 (2006).

[9] Collins DL, Zijdenbos A, Kollokian V, Sled J, Kabani N, Holmes C, Evans A, "Design and construction of a realistic digital brain phantom”, IEEE TMI, 17(3), 463-468 (1998).

[10] Fahn S, Elton RL and members of UPDRS Development Committee, "Unified Parkison’s disease rating scale”, Recent Developments in Parkinson's Disease, 2, 153-163 (1987).

[11] Coupe P, Yger P, Prima S, Hellier P, Kervrann C, Barillot C, "An Optimized Blockwise Non Local Means Denoising Filter for 3D Magnetic Resonance Images”, IEEE TMI, 24(4), 425-441 (2008).

[12] Mangin J-F, “Entropy minimization for automatic correction of intensity non uniformity”, Hilton Head Island, SC:IEEE Press, 162-169 (2000).

[13] Talairach J, Tournoux P, “Co-Planar Stereotaxic Atlas of the Human Brain”, Thieme, Stuttgart, Germany (1988).

[14] Elias WJ, Fu KM, Frysinger RC, “Cortical and subcortical brain shift during stereotactic procedures”, J Neurosurg 107(5), 983-988 (2007).

[15] Miyagi Y, Shima F, Sasaki T, "Brain shift: an error factor during implantation of deep brain stimulation electrodes”, J Neurosurg, 107(5), 989-997 (2007).

[16] Pollo C, Villemure JG, Vingerhoets F, Ghika J, Maeder P, Meuli R, "Magnetic resonance artefact induced by the electrode activa 3389: an in vitro and in vivo study", Acta Neurochirurgica, 146(2), 161-164 (2004).

[17] Pollo C, Vingerhoets F, Pralong E, Ghika J, Maeder P, Meuli R, Thiran J-P, Villemure J-G , "Localization of electrodes in the subthalamic nucleus on magnetic resonance imaging”, J Neurosurg, 106, 36-44 (2007).

[18] Videen TO, Campbell MC, Tabbal SD, Karimi M, Hershey T, Perlmutter JS, "Validation of a fiducial-based atlas localization method for deep brain stimulation contacts in the area of the subthalamic nucleus", $\mathrm{J}$ of Neuroscience methods, 168, 275-281 (2008).

[19] Hemm S, Caire F, Coste J, Vassal F, Nuti C, Derost P, Ouchchane L, Sarry L, Durif F, Lemaire JJ, "Postoperative control in deep brain stimulation of the subthalamic region, the contact membership concept", International Journal of Computer Assisted Radiology and Surgery, 3(1-2), 69-77 (2008).

[20] Sanchez C Javier, "A Cross Validation Study of Deep Brain Stimulation Targeting : From Experts to AtlasBased, Segmentation-Based and Automatic Registration”, IEEE TMI, 25(11), 1440-1450 (2006). 
[21] Wiest-Daesslé, Yger P, Prima S, Barillot C, "Evaluation of a new optimisation algorithm for rigid registration of MRI data”, SPIE Medical Imaging, 6512, 651206 (2007).

[22] Voges J, Volkmann J, Albert N, Lehrke R, Koulousakis A, Freund HJ, Sturm V, "Bilateral high-frequency stimulation in the subthalamic nucleus for the treatment of Parkinson disease: correlation of therapeutic effect with anatomical position”, J Neurosurg, 96(2), 269-279 (2002).

[23] Schaltenbrand G, Wahren W, “Atlas for Stereotaxy of the Human Brain”, Thieme, Stuttgart, Germany (1977).

[24] Seghers D, D'Agostino E, Maes F, Vandermeulen D, Suetens P, “Construction of a Brain Template from MR Images Using State-of-the-Art Registration and Segmentation Techniques”, MICCAI, 696-703 (2004).

[25] Evans AC, Collins DL, Mills SR, Brown ED, Kelly RL, Peters TM, "3D statistical neuroanatomical models from 305 MRI volumes”, Proc IEEE Nucl Science Symp Med Imaging Conf, 1813-1820 (1993).

[26] D’Haese PF, Cetinkaya E, Konrad PE, Kao C, Dawant BM, “Computer-aided placement of deep brain stimulators: From planning to intraoperative”, IEEE TMI, 24(11), 1469-1478 (2005).

[27] Guo T, Parrent AG, Peters TM, "Surgical targeting accuracy analysis of six methods for subthalamic nucleus deep brain stimulation”, Computer Aided Surgery, 12(6), 325-334 (2007).

[28] Lemaire JJ, Coste J, Ouchchane L, Caire F, Nuti C, Derost P, Cristini V, Gabrillargues J, Hemm S, Durif F, Chazal J, "Brain mapping in stereotactic surgery: A brief overview from the probabilistic targeting to the patient-based anatomic mapping”, NeuroImage, 37, S109-S115 (2007).

[29] Dawant B, D’Haese PF, Pallavaram S, Li R, Yu H, Spooner J, Davis T, Kao C, Konrad P, “The VU- DBS project: integrated and computer-assisted planning, intra-operative placement, and post-operative programming of deep-brain stimulators”, SPIE Medical Imaging, 8(28), 65090701-6509710 (2007).

[30] Guo T, Finnis KW, Parrent AG, Peters TM, "Development and Application of Functional Databases for Planning Deep-Brain Neurosurgical Procedures”, MICCAI, 3749, 835-842 (2005).

[31] Holden M, “A review of Geometric Transformation for Nonrigid Body Registration”, IEEE TMI, 27(1), $111-128$ (2008). 